\title{
Solution of the self-dual $\Phi^{4}$ QFT-model on four-dimensional Moyal space ${ }^{1}$
}

\author{
Harald Grosse, ${ }^{a, c}$ Alexander Hock ${ }^{b}$ and Raimar Wulkenhaar ${ }^{b}$ \\ ${ }^{a}$ Fakultät für Physik, Universität Wien, \\ Boltzmanngasse 5, A-1090 Vienna, Austria \\ ${ }^{b}$ Mathematisches Institut der Westfälischen Wilhelms-Universität, \\ Einsteinstraße 62, D-48149 Münster, Germany \\ ${ }^{c}$ Erwin Schrödinger International Institute for Mathematics and Physics, \\ University of Vienna, \\ Boltzmanngasse 9, A-1090 Vienna, Austria \\ E-mail: harald.grosse@univie.ac.at, a_hock03@wwu.de, \\ raimar@math.uni-muenster.de
}

ABSTRACT: Previously the exact solution of the planar sector of the self-dual $\Phi^{4}$-model on 4-dimensional Moyal space was established up to the solution of a Fredholm integral equation. This paper solves, for any coupling constant $\lambda>-\frac{1}{\pi}$, the Fredholm equation in terms of a hypergeometric function and thus completes the construction of the planar sector of the model. We prove that the interacting model has spectral dimension $4-2 \frac{\arcsin (\lambda \pi)}{\pi}$ for $|\lambda|<\frac{1}{\pi}$. It is this dimension drop which for $\lambda>0$ avoids the triviality problem of the matricial $\Phi_{4}^{4}$-model.

We also establish the power series approximation of the Fredholm solution to all orders in $\lambda$. The appearing functions are hyperlogarithms defined by iterated integrals, here of alternating letters 0 and -1 . We identify the renormalisation parameter which gives the same normalisation as the ribbon graph expansion.

KEYwords: Integrable Field Theories, Matrix Models, Non-Commutative Geometry

ArXIV EPRINT: 1908.04543

\footnotetext{
${ }^{1}$ With an appendix by Robert Seiringer, Institute of Science and Technology Austria, Am Campus 1, 3400 Klosterneuburg, Austria.
} 


\section{Contents}

1 Introduction 1

2 Solution via differential equation 3

2.1 Spectral dimension 5

3 Perturbative expansion $\quad 6$

$\begin{array}{lll}3.1 & \text { Recalling earlier results } & 6\end{array}$

3.2 Direct expansion $\quad 7$

$\begin{array}{lll}3.3 & \text { Expansion of the Fredhom equation } & 10\end{array}$

4 Stieltjes transform of the measure function $\quad 13$

$\begin{array}{llr}5 & \text { Outlook } & 14\end{array}$

A On the spectrum of the integral operator $\quad 15$

\section{Introduction}

Many quantum field theory models have been solved or constructed in two dimensions, see e.g. [1-3]. For just-renormalisble bosonic models there is little success so far. The perturbative renormalisation of the $\Phi^{4}$-model on four-dimensional Moyal space with harmonic propagation [4] and the proof that the $\beta$-function vanishes [5] at a self-duality point provided some hope to construct this particular four-dimensional model which is indeed just-renormalisable.

At a special self-duality point [6], the model reduces to a dynamical matrix model with action

$$
S[\Phi]=V \operatorname{Tr}\left(E \Phi^{2}\right)+\frac{\lambda}{4} V \operatorname{Tr}\left(\Phi^{4}\right)
$$

for self-adjoint $\mathcal{N} \times \mathcal{N}$-matrices $\Phi$, where $E$ takes the rôle of a Laplacian whose eigenvalues $E_{n}=\frac{n}{\sqrt{V}}+\frac{\mu_{\text {bare }}^{2}}{2}$ arise with multiplicity $n$. The parameter $V \in \mathbb{R}$ is the deformation parameter of the Moyal space, $\lambda \in \mathbb{R}$ is the coupling constant and $\mu_{\text {bare }}^{2}$ the unrenormalised mass square. The dimension of a field theory is defined by Weyl's Theorem through the asymptotic behavior of the Laplacian, or equivalently by the asymptotic behavior of its corresponding spectral measure. Furthermore, the perturbative expansion of the noncommutative $\Phi^{4}$-model on 4-dimensional Moyal space into Feynman graphs admits the same degrees of divergences for each graph as the ordinary (commutative) $\Phi^{4}$ model in four dimensions. 
The action $S[\Phi]$ is employed to define correlation functions

$$
\left\langle\Phi_{a_{1} b_{1}} \Phi_{a_{2} b_{2}} \ldots \Phi_{a_{n} b_{n}}\right\rangle:=\log \left(\frac{\int \mathrm{d} \Phi \Phi_{a_{1} b_{1}} \Phi_{a_{2} b_{2}} \cdots \Phi_{a_{n} b_{n}} e^{-S[\Phi]}}{\int \mathrm{d} \Phi e^{-S[\Phi]}}\right) .
$$

Integration by parts produces many relations between these correlation functions. Further relations result from a Ward-Takahashi identity discovered in [5]. It was shown in [7] that these relations can be organised into a closed non-linear equation for the planar two-point function and a hierarchy of Dyson-Schwinger equations for all other functions. The latter are linear in the function of interest with an inhomogeneity that only depends on finitely many functions known by induction.

As characteristic to matrix models, the two-point function has a formal genus expansion

$$
\left\langle\Phi_{a b} \Phi_{b a}\right\rangle=\sum_{g=0}^{\infty} V^{1-2 g} Z G_{a b}^{(g)} .
$$

Its planar part $G_{a b}^{(0)}$ can be isolated in a limit $V \rightarrow \infty$. Particularly transparent is a combined limit where also the size $\mathcal{N}$ of the matrices is sent to $\infty$, with the ratio $\frac{\mathcal{N}}{\sqrt{V}}=\Lambda^{2}$ fixed. The previously discrete eigenvalues $E_{n}$ become in this limit functions $E_{x}=x+\frac{\mu_{\text {bare }}^{2}}{2}$ of a real variable $x \in\left[0, \Lambda^{2}\right]$, and $G_{a b}^{(0)}$ converges to $G(x, y)$ with $x=\lim \frac{a}{\sqrt{V}}$ and $y=\lim \frac{b}{\sqrt{V}}$. It this setting the Dyson-Schwinger equation for $G_{a b}^{(0)}$ converges to a non-linear integral equation [7]

$$
\left(\mu_{\text {bare }}^{2}+x+y+\lambda \int_{0}^{\Lambda^{2}} d t t Z G(x, t)\right) Z G(x, y)=1+\lambda \int_{0}^{\Lambda^{2}} d t t Z \frac{G(t, y)-G(x, y)}{t-x} .
$$

It is understood that $\mu_{\text {bare }}^{2}$ and $Z$ depend on the cut-off $\Lambda$. According to the renormalisation philosophy, the task is to determine the precise dependence $\mu_{\text {bare }}^{2}(\Lambda), Z(\Lambda)$ so that the solution $G(x, y)$ of (1.2) has a limit $\Lambda \rightarrow \infty$.

In our recent work [8] we succeeded in solving the analogue of (1.2) for general eigenvalues $E_{a}$ and without requiring the special limit $\mathcal{N}, V \rightarrow \infty$, up to the determination of an implicitly defined measure function. In case of (1.2) this solution specifies to:

Theorem 1 ([8]). Equation (1.2) for the renormalised planar 2-point function of the $\phi^{4}$ QFT-model on four-dimensional noncommutative Moyal space is solved by

$$
\begin{aligned}
G(x, y)=\frac{\mu^{2} \exp (N(x, y))}{\mu^{2}+x+y} & \\
N(x, y):=\frac{1}{2 \pi \mathrm{i}} \int_{-\infty}^{\infty} d t\{ & \log \left(x-J\left(-\frac{\mu^{2}}{2}-\mathrm{i} t\right)\right) \frac{d}{d t} \log \left(y-J\left(-\frac{\mu^{2}}{2}+\mathrm{i} t\right)\right) \\
& -\log \left(-J\left(-\frac{\mu^{2}}{2}-\mathrm{i} t\right)\right) \frac{d}{d t} \log \left(-J\left(-\frac{\mu^{2}}{2}+\mathrm{i} t\right)\right) \\
& -\log \left(x-\left(-\frac{\mu^{2}}{2}-\mathrm{i} t\right)\right) \frac{d}{d t} \log \left(y-\left(-\frac{\mu^{2}}{2}+\mathrm{i} t\right)\right) \\
& \left.+\log \left(-\left(-\frac{\mu^{2}}{2}-\mathrm{i} t\right)\right) \frac{d}{d t} \log \left(-\left(-\frac{\mu^{2}}{2}+\mathrm{i} t\right)\right)\right\},
\end{aligned}
$$


where $J$ is the solution of a Fredholm integral equation of second kind:

$$
J(x)=x-\lambda x^{2} \int_{0}^{\infty} d t \frac{J(t)}{\left(t+\mu^{2}\right)^{2}\left(t+\mu^{2}+x\right)} .
$$

Here $\mu>0$ is a free renormalisation parameter, and $G(0,0)=1$ is already implemented.

As main result of this paper we prove that (1.3) is solved by a hypergeometric function,

$$
J(x)=x_{2} F_{1}\left(\begin{array}{c}
\alpha_{\lambda}, 1-\alpha_{\lambda} \\
2
\end{array} \mid-\frac{x}{\mu^{2}}\right), \quad \text { where } \quad \alpha_{\lambda}:=\frac{\arcsin (\lambda \pi)}{\pi} .
$$

Moreover, we show that the particular choice $\mu^{2}=\frac{\alpha_{\lambda}\left(1-\alpha_{\lambda}\right)}{\lambda}$ provides the same normalisation as the expansion into renormalised ribbon graphs.

The following sections present several methods which we employed to find the solution (1.4) of (1.3). In section 2 we show that a rescaling of $J$ satisfies a hypergeometric differential equation from which we deduce (1.4). Some steps rely on appendix A where the spectrum of an integral operator is determined. In subsection 2.1 we determine the spectral dimension. The treatment via a differential equation is probably the most elegant one. We first obtained this solution via a perturbative expansion described in section 3 . We understand the pattern of the power series solution of (1.3) to $\mathcal{O}\left(\lambda^{10}\right)$ and resum it to (1.4). The advantage of this approach is that it identifies the renormalisation parameter $\mu^{2}$ for which our solution matches the usual perturbative renormalisation prescription. Finally, in section 4 we directly verify (1.4) via integrals for Meijer-G functions.

\section{Solution via differential equation}

It is convenient to symmetrise the Fredholm equation (1.3). Dividing by $\frac{x}{\mu^{2}+x}$ and defining $\tilde{\varrho}_{\lambda}(x):=\frac{J(x)}{x\left(\mu^{2}+x\right)}$, we have

$$
\begin{aligned}
\tilde{\varrho}_{\lambda}(x) & =\frac{1}{\mu^{2}+x}-\lambda \int_{0}^{\infty} d t \frac{\tilde{\varrho}_{\lambda}(t) t x}{\left(\mu^{2}+t\right)\left(\mu^{2}+x\right)\left(\mu^{2}+x+t\right)} \\
& =\frac{c_{\lambda}}{\mu^{2}+x}-\lambda \int_{0}^{\infty} d t \frac{\tilde{\varrho}_{\lambda}(t)}{\mu^{2}+x+t},
\end{aligned}
$$

where $c_{\lambda}=1+\lambda \mu^{2} \int_{0}^{\infty} d t \frac{\tilde{\underline{e}}_{\lambda}(t)}{\mu^{2}+t}=1+\lambda \mu^{2} \int_{0}^{\infty} d t \frac{J(t)}{t\left(\mu^{2}+t\right)^{2}}$. The second line results by (not so obvious) rational fraction expansion. As proved in appendix A, there exists for $\lambda>-\frac{1}{\pi}$ a solution $\tilde{\varrho}_{\lambda} \in L^{2}\left(\mathbb{R}_{+}\right)$, which means $\lim _{t \rightarrow \infty} t \tilde{\varrho}_{\lambda}(t)=0$. Another transformation $\phi(x)=\mu^{2} \tilde{\varrho}_{\lambda}\left(x \mu^{2}\right)$ simplifies the problem to

$$
\phi(x)=\frac{c_{\lambda}}{1+x}-\lambda \int_{0}^{\infty} d t \frac{\phi(t)}{1+t+x}, \quad \phi(0)=1 .
$$

The aim is to find the differential operator $D_{x}$ acting on (2.2) which is reproduced under the integral on $\phi(t)$ such that all appearing inhomogeneous parts vanish, i.e.

$$
D_{x} \phi(x)=-\lambda \int_{0}^{\infty} d t \frac{D_{t} \phi(t)}{1+t+x} .
$$


We compute derivatives and integrate by parts, taking the boundary values at 0 and $\infty$ into account:

$$
\phi^{\prime}(x)=-\frac{c_{\lambda}}{(1+x)^{2}}+\lambda \int_{0}^{\infty} d t \frac{\phi^{\prime}(t)}{1+t+x}+\frac{\lambda}{1+x} .
$$

Also the product with $1+x$ simplifies by integration by parts:

$$
(1+x) \phi^{\prime}(x)=-\frac{c_{\lambda}}{(1+x)}-\lambda \int_{0}^{\infty} d t \frac{t \phi^{\prime}(t)}{1+t+x}
$$

We differentiate once more:

$$
\begin{aligned}
(1+x) \phi^{\prime \prime}(x)+\phi^{\prime}(x) & =\frac{c_{\lambda}}{(1+x)^{2}}+\lambda \int_{0}^{\infty} \frac{d t}{(1+t+x)} \frac{d}{d t}\left(t \phi^{\prime}(t)\right), \\
(1+x) \phi^{\prime \prime}(x) & =\frac{2 c_{\lambda}}{(1+x)^{2}}+\lambda \int_{0}^{\infty} d t \frac{t \phi^{\prime \prime}(t)}{1+t+x}-\frac{\lambda}{1+x} .
\end{aligned}
$$

We multiply by $x$ and integrate by parts:

$$
x(1+x) \phi^{\prime \prime}(x)=\frac{2 c_{\lambda}}{(1+x)}-\frac{2 c_{\lambda}}{(1+x)^{2}}-\lambda \int_{0}^{\infty} \frac{d t t(1+t) \phi^{\prime \prime}(t)}{1+t+x}+\frac{\lambda}{1+x} .
$$

We subtract twice (2.3) and add four times (2.4):

$$
x(1+x) \phi^{\prime \prime}(x)+(2+4 x) \phi^{\prime}(x)=-\frac{2 c_{\lambda}+\lambda}{(1+x)}-\lambda \int_{0}^{\infty} d t \frac{t(1+t) \phi^{\prime \prime}(t)+(2+4 t) \phi^{\prime}(t)}{1+t+x} .
$$

Finally, we add $\frac{2 c_{\lambda}+\lambda}{c_{\lambda}}$ times $(2.2)$ to get $D_{x}=x(1+x) \frac{d^{2}}{d x^{2}}+(2+4 x) \frac{d}{d x}+\frac{2 c_{\lambda}+\lambda}{c_{\lambda}}$, or equivalently

$$
\begin{aligned}
0 & =\left(\mathrm{id}+\lambda \hat{A}_{1}\right) g, \quad \text { where } \\
g(x) & =x(1+x) \phi^{\prime \prime}(x)+(2+4 x) \phi^{\prime}(x)+\frac{2 c_{\lambda}+\lambda}{c_{\lambda}} \phi(x),
\end{aligned}
$$

and $\hat{A}_{\mu}$ is the integral operator with kernel $\hat{A}_{\mu}(t, u)=\frac{1}{u+t+\mu^{2}}$. The arguments given in appendix A show that $\hat{A}_{\mu}$ has spectrum $[0, \pi]$ for any $\mu \geq 0$. Therefore, equation (2.6) has for $\lambda>-\frac{1}{\pi}$ only the trivial solution $g(x)=0$, which is a standard hypergeometric differential equation. The normalisation $\phi(0)=1$ uniquely fixes the solution to

$$
\begin{aligned}
\phi(x) & ={ }_{2} F_{1}\left(\begin{array}{c}
1+\alpha_{\lambda}, 2-\alpha_{\lambda} \\
2
\end{array} \mid-x\right) \\
& =\frac{1}{1+x}{ }_{2} F_{1}\left(\begin{array}{c}
\alpha_{\lambda}, 1-\alpha_{\lambda} \\
2
\end{array} \mid-x\right), \quad c_{\lambda}=\frac{\lambda}{\alpha_{\lambda}\left(1-\alpha_{\lambda}\right)} .
\end{aligned}
$$


It remains to satisfy the boundary condition $c_{\lambda}=1+\lambda \int_{0}^{\infty} d t \frac{\phi(t)}{1+t}$ given after $(2.1)$. The integral can be evaluated via the Euler integral [9, eq. 9.111],

$$
\begin{aligned}
\int_{0}^{\infty} d t \frac{\phi(t)}{1+t} & =\frac{\Gamma(2)}{\Gamma\left(1-\alpha_{\lambda}\right) \Gamma\left(1+\alpha_{\lambda}\right)} \int_{0}^{\infty} d t \int_{0}^{1} d u \frac{u^{-\alpha_{\lambda}}(1-u)^{\alpha_{\lambda}}}{(1+u t)^{\alpha_{\lambda}}(1+t)^{2}} \\
& =\frac{1}{\Gamma\left(1-\alpha_{\lambda}\right) \Gamma\left(1+\alpha_{\lambda}\right)} \int_{0}^{1} d s \int_{0}^{1} d u \frac{u^{-\alpha_{\lambda}}(1-u)^{\alpha_{\lambda}}(1-s)^{\alpha_{\lambda}}}{(1-(1-u) s)^{\alpha_{\lambda}}} \\
& =\frac{1}{\Gamma\left(1-\alpha_{\lambda}\right) \Gamma\left(2+\alpha_{\lambda}\right)} \int_{0}^{1} d u u^{-\alpha_{\lambda}}(1-u)^{\alpha_{\lambda}}{ }_{2} F_{1}\left(\begin{array}{c}
\alpha_{\lambda}, 1 \\
2+\alpha_{\lambda}
\end{array} \mid 1-u\right) \\
& =\frac{1}{\Gamma\left(1-\alpha_{\lambda}\right) \Gamma\left(2+\alpha_{\lambda}\right)} \int_{0}^{1} d u u^{\alpha_{\lambda}}(1-u)^{-\alpha_{\lambda}}\left\{\frac{\left(1+\alpha_{\lambda}\right)}{\alpha_{\lambda}}{ }_{2} F_{1}\left(\begin{array}{c}
\alpha_{\lambda}, 1 \\
1+\alpha_{\lambda}
\end{array} \mid u\right)\right. \\
& =\frac{1}{\Gamma\left(1-\alpha_{\lambda}\right) \Gamma\left(2+\alpha_{\lambda}\right)}\left\{\frac{\left(1+\alpha_{\lambda}\right)}{\alpha_{\lambda}} F_{1}\left(\begin{array}{c}
\alpha_{\lambda}, 2 \\
2+\alpha_{\lambda}
\end{array} \mid u\right)\right\} \\
& =\frac{1}{\alpha_{\lambda}} \frac{\left.1+\alpha_{\lambda}\right) \Gamma\left(1-\alpha_{\lambda}\right) \Gamma\left(1-\alpha_{\lambda}\right)}{\Gamma\left(2-\alpha_{\lambda}\right) \Gamma(1)} \\
\alpha_{\lambda}\left(1-\alpha_{\lambda}\right) & \left.\frac{\Gamma\left(2+\alpha_{\lambda}\right) \Gamma\left(1+\alpha_{\lambda}\right) \Gamma\left(1-\alpha_{\lambda}\right) \Gamma\left(1-\alpha_{\lambda}\right)}{\Gamma(2) \Gamma(2) \Gamma(1)}\right\}
\end{aligned}
$$

Here we have transformed $t=\frac{s}{1-s}$, evaluated first the $s$-integral [9, eq. 9.111] to a hypergeometric function, used its contiguous relation [9, eq. 9.137.17] so that the remaining integrals are known from [9, eq. 7.512.4] and [9, eq. 7.512.3]. We thus conclude

$$
c_{\lambda}=1+\frac{\lambda}{\alpha_{\lambda}\left(1-\alpha_{\lambda}\right)}-\frac{\lambda \pi}{\sin \left(\alpha_{\lambda} \pi\right)} \stackrel{!}{=} \frac{\lambda}{\alpha_{\lambda}\left(1-\alpha_{\lambda}\right)}
$$

with solution

$$
\sin \left(\alpha_{\lambda} \pi\right)=\lambda \pi, \quad \alpha_{\lambda}=\left\{\begin{array}{c}
\frac{\arcsin (\lambda \pi)}{\pi} \quad \text { for }|\lambda| \leq \frac{1}{\pi} \\
\frac{1}{2}+\mathrm{i} \frac{\operatorname{arcosh}(\lambda \pi)}{\pi} \text { for } \lambda \geq \frac{1}{\pi}
\end{array}\right.
$$

The branch is uniquely selected by the requirement $\lim _{\lambda \rightarrow 0} c_{\lambda}=1$. For $\lambda<-\frac{1}{\pi}$ there is no solution for which $c_{\lambda}$ and $\phi$ are real. Transforming back to $\tilde{\rho}_{\lambda}$ and $J$ gives the result announced in (1.4), which provides the two-point function $G(x, y)$ via Thm. 1.

\subsection{Spectral dimension}

Let $\varrho_{0}(x) d x$ be the spectral measure of the operator $E$ in the initial action (1.1). The main discovery of [8] was that the interaction $\frac{\lambda}{4} \operatorname{Tr}\left(\Phi^{4}\right)$ effectively modifies the spectral measure to $\varrho_{\lambda}(x) d x$. What before, when expressed in terms of $\varrho_{0}(x) d x$, was intractable became suddenly exactly solvable in terms of the deformation $\varrho_{\lambda}(x) d x$. For four-dimensional Moyal space one has $\varrho_{0}(x)=x$ and $\varrho_{\lambda}(x)=J(x)$. The explicit solution (1.4) shows that the deformation is drastic: it changes the spectral dimension $D$ defined by $D=$ $\inf \left\{p: \int_{0}^{\infty} d t \frac{\varrho_{\lambda}(t)}{(1+t)^{p / 2}}<\infty\right\}$. 
Lemma 2.1. For any $\left|\alpha_{\lambda}\right|<\frac{1}{2}$ one has

$$
\frac{1}{(1+x)^{\alpha_{\lambda}}} \leq{ }_{2} F_{1}\left(\begin{array}{c}
\alpha_{\lambda}, 1-\alpha_{\lambda} \\
2
\end{array} \mid-x\right) \leq \frac{\Gamma\left(1-2 \alpha_{\lambda}\right)}{\Gamma\left(2-\alpha_{\lambda}\right) \Gamma\left(1-\alpha_{\lambda}\right)} \frac{1}{(1+x)^{\alpha_{\lambda}}} .
$$

Proof. We transform with [9, eq. 9.131.1] to

$$
{ }_{2} F_{1}\left(\begin{array}{c}
\alpha_{\lambda}, 1-\alpha_{\lambda} \\
2
\end{array} \mid-x\right)=\left(\frac{1}{1+x}\right)^{\alpha_{\lambda}} \frac{{ }_{2} F_{1}\left(\begin{array}{c}
2-\alpha_{\lambda}, 1-\alpha_{\lambda} \\
2
\end{array} \mid \frac{x}{1+x}\right)}{\left(1-\frac{x}{1+x}\right)^{2 \alpha_{\lambda}-1}}
$$

By [10, Thm. 1.10], the fraction on the r.h.s. is strictly increasing from 1 at $x=0$ to its limit $\frac{B\left(2,1-2 \alpha_{\lambda}\right)}{B\left(2-\alpha_{\lambda}, 1-\alpha_{\lambda}\right)}=\frac{\Gamma\left(1-2 \alpha_{\lambda}\right)}{\Gamma\left(2-\alpha_{\lambda}\right) \Gamma\left(1-\alpha_{\lambda}\right)}$ for $x \rightarrow \infty$.

Corollary 2.2. For $|\lambda|<\frac{1}{\pi}$, the deformed measure $\varrho_{\lambda}=J$ of four-dimensional Moyal space has spectral dimension $D=4-2 \frac{\arcsin (\lambda \pi)}{\pi}$.

Proof. Lemma 2.1 together with $\varrho_{\lambda}(x)=J(x)$ and (1.4) gives the assertion.

The change of spectral dimension is important. If instead of (1.3) the function $J$ was given by $\tilde{J}(x)=x-\lambda x^{2} \int_{0}^{\infty} d t \frac{\varrho_{0}(t)}{\left(t+\mu^{2}\right)^{2}\left(t+\mu^{2}+x\right)}$, then for $\varrho_{0}(x)=x$ this function $\tilde{J}$ is bounded above. Hence, $\tilde{J}^{-1}$ needed in higher topological sectors could not exist globally on $\mathbb{R}_{+}$, which would render the model inconsistent for any $\lambda>0$. The dimension drop down to $D=4-2 \frac{\arcsin (\lambda \pi)}{\pi}$ avoids this (triviality) problem.

\section{Perturbative expansion}

In this section we study two different perturbative expansions of an angle function which is behind the solution of $G(x, y)$. In section 3.2 we directly expand (3.2) order by order in $\lambda$, whereas in section 3.3 we expand (1.3) and compare with the other result via Corollary 3.1. For a special choice of $\mu^{2}$ which we determine, both expansions coincide order by order in $\lambda$ (we played the game up to the $10^{\text {th }}$ order with a computer algebra system).

\subsection{Recalling earlier results}

Equation (1.2) is a nonlinear singular integral equation. The solution theory for linear integral equations of Carleman type is known (see e.g. [11]) and suggests the ansatz

$$
G(a, b)=\frac{\sin \left(\tau_{b}(a)\right)}{\lambda \pi a} e^{\mathcal{H}_{a}^{\Lambda}\left[\tau_{b}(\bullet)\right]-\mathcal{H}_{0}^{\Lambda}\left[\tau_{0}(\bullet)\right]},
$$

where $\mathcal{H}_{a}^{\Lambda}[f(\bullet)]:=\frac{1}{\pi} \lim _{\varepsilon \rightarrow 0}\left(\int_{0}^{a-\varepsilon}+\int_{a+\varepsilon}^{\Lambda^{2}}\right) d p \frac{f(p)}{p-a}$ denotes the finite Hilbert transform. Inserting (3.1) into (1.2) gives with identities established in [12] the consistency relation

$$
p \lambda \pi \cot \left(\tau_{a}(p)\right)=\mu_{\text {bare }}^{2}+a+p+\lambda \pi \mathcal{H}_{p}^{\Lambda}[\bullet]+\frac{1}{\pi} \int_{0}^{\Lambda^{2}} d t \tau_{p}(t)
$$


Renormalisation by Taylor subtraction at 0 suggests to choose the bare mass according to

$$
\mu_{\text {bare }}^{2}=1-\lambda \Lambda^{2}-\frac{1}{\pi} \int_{0}^{\Lambda^{2}} d t \tau_{0}(t)
$$

We will later see that another form of (3.3) is for the exact solution more efficient.

The key step in [8] to solve (3.2) (actually in larger generality) was to define a $\lambda$ deformation $\varrho_{\lambda}(x)$ of a spectral measure function $\varrho_{0}$. This deformed measure then gives rise to a function $J(x)$ which in four dimensions reads

$$
J(z):=z-\lambda z^{2} \int_{0}^{\infty} d t \frac{\varrho_{\lambda}(t)}{\left(t+\mu^{2}\right)^{2}\left(t+\mu^{2}+z\right)} .
$$

The system of functions $\left(\varrho_{0}, \varrho_{\lambda}, J\right)$ is closed by the final condition $\varrho_{0}(J(x))=\varrho_{\lambda}(x)$.

In general this is a complicated system of equations. Here, the integral equation (1.2) encodes the spectral measure $\varrho_{0}(x)=x$ so that $J(x)=\varrho_{\lambda}(x)$ and (3.4) is reduced to (1.3). We now have the following corollary of [8, Thm. 2.7]:

Corollary 3.1. Adjusting the bare mass to

$$
\mu_{\text {bare }}^{2}(\Lambda)=\mu^{2} \cdot\left(1-\lambda \int_{0}^{J^{-1}\left(\Lambda^{2}\right)} d t \frac{\varrho_{\lambda}(t)}{\left(t+\mu^{2}\right)^{2}}\right)-2 \lambda \int_{0}^{J^{-1}(\Lambda)} d t \frac{\varrho_{\lambda}(t)}{\left(t+\mu^{2}\right)}
$$

then the consistency relation (3.2) is solved by

$$
\begin{aligned}
& \lambda \pi \varrho_{0}(p) \cot \left(\tau_{a}(p)\right)=\lim _{\varepsilon \rightarrow 0} \operatorname{Re}(a+I(p+\mathrm{i} \varepsilon)), \\
& \text { where } \quad I(z):=-J\left(-\mu^{2}-J^{-1}(z)\right) .
\end{aligned}
$$

Note that (3.5) fixes the renormalisation different than (3.3). It is actually a family of renormalisations which depend on a free parameter $\mu^{2}(\lambda)$. Setting $G(0,0)=1$ does not mean $\mu^{2}=1$, nevertheless both approaches coincide in the limit $\Lambda^{2} \rightarrow \infty$. We will later identify this unique function $\mu^{2}(\lambda)$ that gives (3.3).

\subsection{Direct expansion}

Expanding equation (3.2) with renormalisation (3.3) and finite cut-off gives

$$
p \lambda \pi \cot \left(\tau_{a}(p)\right)=1+a+p+\lambda p \log \left(\frac{\Lambda^{2}-p}{p}\right)+\frac{1}{\pi} \int_{0}^{\Lambda^{2}} d t\left(\tau_{p}(t)-\tau_{0}(t)\right) .
$$

The first order is extracted directly

$$
p \lambda \pi \cot \left(\tau_{a}(p)\right)=1+a+p+\mathcal{O}\left(\lambda^{1}\right) \quad \Rightarrow \quad \tau_{a}(p)=\frac{p \lambda \pi}{1+a+p}+\mathcal{O}\left(\lambda^{2}\right),
$$

which gives after inserting back at the next order

$$
\begin{aligned}
p \lambda \pi \cot \left(\tau_{a}(p)\right)=1+a+p+\lambda( & (1+p) \log (1+p)-p \log (p) \\
& \left.+p \log \left(\frac{\Lambda^{2}-p}{1+p+\Lambda^{2}}\right)+\log \left(\frac{1+\Lambda^{2}}{1+p+\Lambda^{2}}\right)\right)+\mathcal{O}\left(\lambda^{2}\right) .
\end{aligned}
$$


The limit $\Lambda^{2} \rightarrow \infty$ gives finite results for $\cot \left(\tau_{a}(p)\right)$ as well as for $\tau_{a}(p)$ order by order, however the limit has to be taken with caution. Integral and limit do not commute. Namely, for and expansion $\tau_{a}(p)=\sum_{n=1}^{\infty} \lambda^{n} \tau_{a}^{(n)}(p)$ we have

$$
\lim _{\Lambda^{2} \rightarrow \infty} \int_{0}^{\Lambda^{2}} d t\left(\tau_{p}^{(n)}(t)-\tau_{0}^{(n)}(t)\right) \neq \int_{0}^{\infty} d t \lim _{\Lambda^{2} \rightarrow \infty}\left(\tau_{p}^{(n)}(t)-\tau_{0}^{(n)}(t)\right), \quad n>1 .
$$

As an example we will look at the next order of both integrals. They give

$$
\begin{aligned}
& \lim _{\Lambda^{2} \rightarrow \infty} \frac{1}{\pi} \int_{0}^{\Lambda^{2}} d t\left(\tau_{p}^{(2)}(t)-\tau_{0}^{(2)}(t)\right) \\
& =(1+p) \log (1+p)^{2}+(1+2 p) \operatorname{Li}_{2}(-p)-p \zeta_{2}, \\
& \frac{1}{\pi} \int_{0}^{\infty} d t \lim _{\Lambda^{2} \rightarrow \infty}\left(\tau_{p}^{(2)}(t)-\tau_{0}^{(2)}(t)\right) \\
& =\int_{0}^{\infty} d t t\left(\frac{t \log (t)-(1+t) \log (1+t)}{(1+t+p)^{2}}-\frac{t \log (t)-(1+t) \log (1+t)}{(1+t)^{2}}\right) \\
& =(1+p) \log (1+p)^{2}+(1+2 p) \operatorname{Li}_{2}(-p)+2 p \zeta_{2},
\end{aligned}
$$

respectively, where $\operatorname{Li}_{n}(x)$ is the $n^{\text {th }}$ polylogarithm and $\zeta_{n} \equiv \zeta(n)$ is the Riemann zeta value at integer $n$. The last term makes the difference. Taking the "wrong" second result and plugging it back into (3.7) would lead to divergences at the next order. Consequently, we have to treat the perturbative expansion of (3.7) with a finite cut-off $\Lambda^{2}$ at all orders, where each order has a finite limit.

The integration theory of the appearing integrals is completely understood in form of iterated integrals [13]. They form a shuffle algebra, which is symbolically implemented in the Maple package HyPERInt [14].

We computed the first 6 orders via HyperInt for finite $\Lambda^{2}$. Sending $\Lambda^{2} \rightarrow \infty$ is well-defined at any order as expected. The first orders read explicitly

$$
\begin{array}{rl}
\lim _{\Lambda^{2} \rightarrow \infty} p \lambda \pi \cot \left(\tau_{a}(p)\right)=1 & a+p+\lambda((1+p) \log (1+p)-p \log (p)) \\
+ & \lambda^{2}\left(-p \zeta_{2}+(1+p) \log (1+p)^{2}+(1+2 p) \operatorname{Li}_{2}(-p)\right) \\
+ & \lambda^{3}\left(\zeta_{2} \log (1+p)-\frac{1+p}{2 p} \log (1+p)^{2}+(1+p) \log (1+p)^{3}\right. \\
& +2 p \zeta_{3}-2 p \operatorname{Li}_{3}(-p)-(1+2 p) \operatorname{Hlog}(p,[-1,0,-1]) \\
& -2(2+3 p) \operatorname{Hlog}(p,[0,-1,-1]))+\mathcal{O}\left(\lambda^{4}\right) .
\end{array}
$$

The hyperlogarithms Hlog are defined by the iterated integrals

$$
\operatorname{Hlog}\left(a,\left[k_{1}, \ldots, k_{n}\right]\right):=\int_{0}^{a} \frac{d x_{1}}{x_{1}-k_{1}} \int_{0}^{x_{1}} \frac{d x_{2}}{x_{2}-k_{2}} \ldots \int_{0}^{x_{n-1}} \frac{d x_{n}}{x_{n}-k_{n}},
$$

where the $k_{i}$ are called letters. An alternative notation is $\operatorname{Hlog}\left(a,\left[k_{1}, \ldots, k_{n}\right]\right)=$ $L_{k_{1}, \ldots, k_{n}}(a)$. Important special cases $\operatorname{are} \operatorname{Hlog}(a,[\underbrace{-k, \ldots,-k}_{n}])=\frac{\log \left(1+\frac{a}{k}\right)^{n}}{n !}$ for $k \in \mathbb{N}^{\times}$, $\operatorname{Hlog}(a,[\underbrace{0, \ldots, 0}_{n}]):=\frac{\log (a)^{n}}{n !}$ and $\operatorname{Hlog}(a,[\underbrace{0, \ldots, 0}_{n},-1])=-\operatorname{Li}_{1+n}(-a)$. 
The perturbative expansion shows that the branch point at $p=-1$ plays an important role. Its boundary value is found to be $\lim _{\substack{\Lambda^{2} \rightarrow \infty \\ \varepsilon \searrow 0}} \cot \left(\tau_{0}(-1+\mathrm{i} \varepsilon)\right)=-\mathrm{i}+\mathcal{O}\left(\lambda^{7}\right)$. It is natural to conjecture that it holds at any order,

$$
\lim _{\substack{\Lambda_{\varepsilon \rightarrow \infty}^{2} \rightarrow 0 \\ \varepsilon \searrow 0}} \cot \left(\tau_{0}(-1+\mathrm{i} \varepsilon)\right)=-\mathrm{i}
$$

The perturbative expansion with a finite cut-off $\Lambda^{2}$ is quite inefficient. The boundary value (3.9) admits a more efficient strategy. We take the derivative of (3.7) with respect to $p$ :

$$
1+\lambda \log \left(\frac{\Lambda^{2}-p}{p}\right)-\lambda \frac{\Lambda^{2}}{\Lambda^{2}-p}+\frac{1}{\pi} \int_{0}^{\Lambda^{2}} d t \frac{d \tau_{p}(t)}{d p}=\lambda \pi \cot \left(\tau_{a}(p)\right)+p \lambda \pi \frac{\partial}{\partial p} \cot \left(\tau_{a}(p)\right) .
$$

Multiplying this equation by $p$ and subtracting it from (3.7) again leads to

$$
-p^{2} \lambda \pi \frac{\partial}{\partial p} \cot \left(\tau_{a}(p)\right)=1+a+\lambda \frac{p \Lambda^{2}}{\Lambda^{2}-p}+\frac{1}{\pi} \int_{0}^{\Lambda^{2}} d t\left(\tau_{p}(t)-\tau_{0}(t)-p \frac{d \tau_{p}(t)}{d p}\right),
$$

where the limit $\Lambda^{2} \rightarrow \infty$ is now safe from the beginning and commutes with the integral. We divide (3.10) by $-p^{2}$ and integrate it for all orders higher than $\lambda^{1}$ over $p$ from -1 (here (3.9) is assumed) up to some $q$ to get $\lim _{\Lambda^{2} \rightarrow \infty} \lambda \pi \cot \left(\tau_{a}(q)\right)$ on the l.h.s. On the r.h.s. the order of integrals $\int_{-1}^{q} d p \int_{0}^{\infty} d t$ can be exchanged. The integral over $p$ is

$$
\int_{-1}^{q} d p \frac{1}{p^{2}}\left(\tau_{p}(t)-\tau_{0}(t)-p \frac{d \tau_{p}(t)}{d p}\right)
$$

assuming Hölder continuity of $\tau_{p}(t)$ so that the integral splits after taking principal values. The last term is computed for small $\epsilon$ and all $\mathcal{O}\left(\lambda^{>1}\right)$-contributions via integration by parts

$$
\begin{aligned}
\int_{[-1, q] \backslash[-\epsilon, \epsilon]} d p \frac{\frac{d \tau_{p}(t)}{d p}}{p} & =\left.\frac{\tau_{p}(t)}{p}\right|_{p=\epsilon} ^{q}+\left.\frac{\tau_{p}(t)}{p}\right|_{p=-1} ^{-\epsilon}+\int_{[-1, q] \backslash[-\epsilon, \epsilon]} d p \frac{\tau_{p}(t)}{p^{2}} \\
& =\frac{\tau_{q}(t)}{q}+\tau_{-1}(t)+\int_{[-1, q] \backslash[-\epsilon, \epsilon]} d p \frac{\tau_{p}(t)}{p^{2}}-\frac{\tau_{-\epsilon}(t)+\tau_{\epsilon}(t)}{\epsilon} .
\end{aligned}
$$

The first term in (3.11) cancels. The second term in (3.11) integrates to a boundary term $+2 \frac{\tau_{0}(t)}{\epsilon}$, which is also canceled by the last term of (3.12). Multiplying by $q$ and including the special $\mathcal{O}(\lambda)$-contribution we arrive in the limit $\Lambda^{2} \rightarrow \infty$ where (3.9) is (conjecturally) available at

$$
q \lambda \pi \cot \left(\tau_{a}(q)\right)=1+a+q-\lambda q \log (q)+\frac{1}{\pi} \int_{0}^{\infty} d t\left(\tau_{q}(t)-(1+q) \tau_{0}(t)+q \tau_{-1}(t)\right) .
$$

This equation is much more appropriate for the perturbation theory because the number of terms is reduced tremendously order by order. Obviously, the first six order coincide with the earlier but much harder perturbative expansion of (3.7).

Using (3.13) the perturbative expansion is increased up to $\lambda^{9}$ with HyPERInT. As consistency check of assumption (3.9) we inserted the next orders $\tau_{a}^{(n)}(p)$ into (3.1) to get the expansion $G(a, b)=\sum_{n=0}^{\infty} \lambda^{n} G^{(n)}(a, b)$. This confirmed the symmetry $G^{(n)}(a, b)=$ $G^{(n)}(b, a)$ which would easily be lost by wrong assumptions. We are thus convinced to have the correct expressions for $\tau_{a}^{(n)}(p)$ for $6<n<10$. 


\subsection{Expansion of the Fredhom equation}

To access the angle function $\tau_{a}(p)$ through Corollary 3.1 we first have to determine the expansion of the deformed measure $\varrho_{\lambda}(x)=J(x)$ through the Fredholm equation (1.3). The constant $\mu^{2}(\lambda)$ is not yet fixed and needs a further expansion

$$
\mu^{2}=\sum_{n=0}^{\infty} \lambda^{n} \mu_{n}^{2} .
$$

First orders of the deformed measure are given iteratively through (1.3)

$$
\begin{aligned}
\varrho_{\lambda}(x)= & x-\lambda\left(\left(x+\mu_{0}^{2}\right) \operatorname{Hlog}\left(t,\left[-\mu_{0}^{2}\right]\right)-x\right) \\
& -\frac{\lambda^{2}}{\mu_{0}^{2}}\left(-\mu_{0}^{2} x \operatorname{Hlog}\left(x,\left[0,-\mu_{0}^{2}\right]\right)+\mu_{0}^{2}\left(\mu_{1}^{2}+\mu_{0}^{2}+x\right) \operatorname{Hlog}\left(x,\left[-\mu_{0}^{2}\right]\right)-x\left(\mu_{1}^{2}+\mu_{0}^{2}\right)\right) \\
& +\mathcal{O}\left(\lambda^{3}\right) .
\end{aligned}
$$

Recall that the inverse of $J(x)=\varrho_{\lambda}(x)=p$ exists for all $p \in \mathbb{R}_{+}$in case $\lambda<\left(\int_{0}^{\infty} \frac{d t \varrho_{\lambda}(t)}{\left(t+\mu^{2}\right)^{2}}\right)^{-1}$. If $\varrho_{\lambda}(x)$ had the same asymptotics as $\varrho_{0}(x)=x$ then $J^{-1}$ could not be defined globally for $\lambda>0$. We proved in section 2.1 that the asymptotics of $\varrho_{\lambda}(x)$ is altered in such a way that $J^{-1}$ is defined. Anyway, in each order of perturbative expansion the inverse $J^{-1}$ is globally defined on $\mathbb{R}_{+}$. At this point it suffices to assume that $J^{-1}(p)$ is a formal power series in $\lambda$, which is achieved by (3.4)

$$
J^{-1}(p)=p+\lambda\left(J^{-1}(p)\right)^{2} \int_{0}^{\infty} \frac{d t \varrho_{\lambda}(t)}{\left(t+\mu^{2}\right)^{2}\left(t+\mu^{2}+J^{-1}(p)\right)} .
$$

Expanding $\varrho_{\lambda}(t)$ and $\mu^{2}$, the first orders are

$$
\begin{aligned}
J^{-1}(p)= & p-\lambda\left(p-\left(\mu_{0}^{2}+p\right) \operatorname{Hlog}\left(p,\left[-\mu_{0}^{2}\right]\right)\right) \\
& -\frac{\lambda^{2}}{\mu_{0}^{2}}\left(p \mu_{0}^{2} \operatorname{Hlog}\left(p,\left[0,-\mu_{0}^{2}\right]\right)-2 \mu_{0}^{2}\left(p+\mu_{0}^{2}\right) \operatorname{Hlog}\left(p,\left[-\mu_{0}^{2},-\mu_{0}^{2}\right]\right)\right. \\
& \left.-\mu_{0}^{2}\left(\mu_{1}^{2}+\mu_{0}^{2}\right) \operatorname{Hlog}\left(p,\left[-\mu_{0}^{2}\right]\right)+p\left(\mu_{1}^{2}+\mu_{0}^{2}\right)\right)+\mathcal{O}\left(\lambda^{3}\right) .
\end{aligned}
$$

The last step is to determine $\lim _{\varepsilon \rightarrow 0} \operatorname{Re} I(p+i \varepsilon)=p \lambda \pi \cot \left(\tau_{0}(p)\right)$ for $\Lambda^{2} \rightarrow \infty$ via

$$
I(z)=\mu^{2}+J^{-1}(z)+\lambda\left(\mu^{2}+J^{-1}(z)\right)^{2} \int_{0}^{\infty} \frac{d t \varrho_{\lambda}(t)}{\left(t+\mu^{2}\right)^{2}\left(t-J^{-1}(z)\right)},
$$

as a formal series. The first few orders are

$$
\begin{aligned}
\lim _{\varepsilon \rightarrow 0} I(p+i \varepsilon)= & \mu_{0}^{2}+p+\lambda\left(\mathrm{i} \pi p+\mu_{0}^{2}+\mu_{1}^{2}+\left(\mu_{0}^{2}+p\right) \operatorname{Hlog}\left(p,\left[-\mu_{0}^{2}\right]\right)+p \log \left(\mu_{0}^{2}\right)-p \log (p)\right) \\
& +\lambda^{2}\left(\mu_{0}^{2}\left(1-\zeta_{2}\right)+\mu_{1}^{2}+\mu_{2}^{2}-p \zeta_{2}+\left(\mu_{0}^{2}+\mu_{1}^{2}\right) \operatorname{Hlog}\left(p,\left[-\mu_{0}^{2}\right]\right)\right. \\
& \left.+2\left(\mu_{0}^{2}+p\right) \operatorname{Hlog}\left(p,\left[-\mu_{0}^{2},-\mu_{0}^{2}\right]\right)-\left(\mu_{0}^{2}+2 p\right) \operatorname{Hlog}\left(p,\left[0,-\mu_{0}^{2}\right]\right)\right)+\mathcal{O}\left(\lambda^{3}\right) .
\end{aligned}
$$

Comparing it with (3.8) through equation (3.6) fixes every $\mu_{i}^{2}$ uniquely and confirms

$$
\lim _{\varepsilon \rightarrow 0} I(p+\mathrm{i} \varepsilon)=\lambda \pi p \cot \left(\tau_{0}(p)\right)+\mathrm{i} \lambda \pi p .
$$


Furthermore, the first 10 orders are identical with the expansion of (3.13), provided that the $\mu_{i}^{2}$ 's are fixed to

$$
\begin{aligned}
\mu^{2}= & 1-\lambda+\frac{1}{6}(\pi \lambda)^{2}-\lambda \frac{1}{3}(\pi \lambda)^{2}+\frac{3}{40}(\pi \lambda)^{4}-\lambda \frac{8}{45}(\pi \lambda)^{4}+\frac{5}{112}(\pi \lambda)^{6}-\lambda \frac{4}{35}(\pi \lambda)^{6} \\
& +\frac{35}{1152}(\pi \lambda)^{8}-\lambda \frac{128}{1575}(\pi \lambda)^{8}+\frac{63}{2816}(\pi \lambda)^{10}+\mathcal{O}\left(\lambda^{11}\right) .
\end{aligned}
$$

The conjectured behavior of $\cot \left(\tau_{0}(p)\right)$ at $p=-1+\mathrm{i} \varepsilon$ in the previous subsection (3.9) is now equivalent to

$$
\lim _{\varepsilon \rightarrow 0} I(-1+\mathrm{i} \varepsilon)=0 \quad \Rightarrow \quad J^{-1}(-1)=-\mu^{2} .
$$

We find that the expansion (3.14) of $\mu^{2}$ obeys an unexpected boundary condition

$$
\int_{0}^{\infty} \frac{d t \varrho_{\lambda}(t)}{\left(\mu^{2}+t\right)^{3}}=\frac{1}{2}+\mathcal{O}\left(\lambda^{10}\right)
$$

For further study we pass as in section 2 to the rescaled measure $\phi(x)=\mu^{2} \tilde{\varrho}_{\lambda}\left(\mu^{2} x\right):=$ $\frac{\varrho_{\lambda}\left(\mu^{2} x\right)}{\mu^{2} x(1+x)}$. The pattern of coefficients of the $\mu^{2}$-expansion in (3.14) suggests to distinguish between even an odd powers in $\lambda$. The even powers $\lambda^{2 n}$ are given by the formula

$$
\frac{(2 n-1) ! !}{(2 n) ! !(2 n+1)}=\frac{(2 n) !}{4^{n} n !^{2}(2 n+1)}
$$

and the odd powers $\lambda^{2 n+1}$ by

$$
2 \frac{(2 n) ! !}{(2 n+1) ! !(2 n+2)}=2 \frac{4^{n} n !^{2}}{(2 n+2) !}
$$

Both series are convergent for $|\lambda|<\frac{1}{\pi}$ with the result (up to order $\lambda^{10}$ )

$$
\mu^{2}=\frac{\arcsin (\lambda \pi)}{\lambda \pi}-\lambda\left(\frac{\arcsin (\lambda \pi)}{\lambda \pi}\right)^{2}
$$

This result suggests that $\frac{\arcsin (\lambda \pi)}{\pi}$ is a better expansion parameter than $\lambda$ itself. The factors $\pi^{2 n}$ are produced by $\zeta_{2 n}$ in the iterated integrals. We thus reorganise the perturbative solution of (2.2) into a series in $\frac{\arcsin (\lambda \pi)}{\pi}$. The power of $\frac{\arcsin (\lambda \pi)}{\lambda \pi}$ depends on the number of letters of the hyperpolylogarithm, which alternate between -1 and 0 . The expansion which holds up to order $\lambda^{10}$ is given by

$$
\begin{aligned}
\phi(x)= & c_{\lambda} \frac{\arcsin (\lambda \pi)}{\lambda \pi(1+x)} \sum_{n=0}^{\infty} \operatorname{Hlog}(x,[\underbrace{0,-1, \ldots, 0,-1}_{n}])\left(\frac{\arcsin (\lambda \pi)}{\pi}\right)^{2 n} \\
& -\lambda c_{\lambda} \frac{\arcsin (\lambda \pi)^{2}}{x(\lambda \pi)^{2}} \sum_{n=0}^{\infty} \operatorname{Hlog}(x,[-1, \underbrace{0,-1, \ldots, 0,-1}_{n}])\left(\frac{\arcsin (\lambda \pi)}{\pi}\right)^{2 n},
\end{aligned}
$$

where the underbrace with $n$ means that we have $n$ times the letters 0 and -1 in an alternating way. 
In the limit $x \rightarrow 0$ only the terms with $n=0$ in both sums survive,

$$
\begin{aligned}
1 \equiv \phi(0) & =c_{\lambda} \frac{\arcsin (\lambda \pi)}{\lambda \pi} \lim _{x \rightarrow 0} \frac{\operatorname{Hlog}(x,[])}{1+x}-\lambda c_{\lambda} \frac{\arcsin (\lambda \pi)^{2}}{(\lambda \pi)^{2}} \lim _{x \rightarrow 0} \frac{\operatorname{Hlog}(x,[-1])}{x} \\
& =\frac{c_{\lambda}}{\lambda} \frac{\arcsin (\lambda \pi)}{\pi}\left(1-\frac{\arcsin (\lambda \pi)}{\lambda \pi}\right) .
\end{aligned}
$$

This value was found in section 2 by another method. We also remark that $c_{\lambda}=\frac{1}{\mu^{2}}$ for the special renormalisation.

Next define the functions

$$
\begin{aligned}
& f(x):=\sum_{n=0}^{\infty} \operatorname{Hlog}(x,[\underbrace{0,-1, \ldots, 0,-1}_{n}]) \alpha_{\lambda}^{2 n} \\
& g(x):=\sum_{n=0}^{\infty} \operatorname{Hlog}(x,[-1, \underbrace{0,-1, \ldots, 0,-1}_{n}]) \alpha_{\lambda}^{2 n},
\end{aligned}
$$

where $\alpha_{\lambda}=\frac{\arcsin (\lambda \pi)}{\pi}$. Both together obey the differential equations

$$
f^{\prime}(x)=\frac{\alpha_{\lambda}^{2}}{x} g(x) \quad g^{\prime}(x)=\frac{1}{1+x} f(x),
$$

or equivalently

$$
f^{\prime \prime}(x)+\frac{f^{\prime}(x)}{x}-\alpha_{\lambda}^{2} \frac{f(x)}{(1+x) x}=0, \quad g^{\prime \prime}(x)+\frac{g^{\prime}(x)}{1+x}-\alpha_{\lambda}^{2} \frac{g(x)}{(1+x) x}=0,
$$

with the boundary conditions $f(0)=1, f^{\prime}(0)=\alpha_{\lambda}^{2}, g(0)=0$ and $g^{\prime}(0)=1$. The solution is given by hypergeometric functions ${ }_{2} F_{1}$

$$
f(x)={ }_{2} F_{1}\left(\begin{array}{c}
\alpha_{\lambda},-\alpha_{\lambda} \\
1
\end{array} \mid-x\right) \quad g(x)=\frac{x}{\alpha_{\lambda}^{2}} f^{\prime}(x)=x_{2} F_{1}\left(\begin{array}{c}
1+\alpha_{\lambda}, 1-\alpha_{\lambda} \\
2
\end{array} \mid-x\right) .
$$

In summary, the solution of equation (2.2) is conjectured to be

$$
\begin{aligned}
\phi(x) & =\frac{\alpha_{\lambda} c_{\lambda}}{\lambda(1+x)}{ }_{2} F_{1}\left(\begin{array}{c}
\alpha_{\lambda},-\alpha_{\lambda} \\
1
\end{array} \mid-x\right)-\frac{\alpha_{\lambda}^{2} c}{\lambda}{ }_{2} F_{1}\left(\begin{array}{c}
1+\alpha_{\lambda}, 1-\alpha_{\lambda} \\
2
\end{array} \mid-x\right) \\
& ={ }_{2} F_{1}\left(\begin{array}{c}
1+\alpha_{\lambda}, 2-\alpha_{\lambda} \\
2
\end{array} \mid-x\right)
\end{aligned}
$$

or equivalently for (1.3)

$$
J(x)=\varrho_{\lambda}(x)=\frac{x}{\mu^{2}}\left(1+\frac{x}{\mu^{2}}\right) f\left(\frac{x}{\mu^{2}}\right)=x_{2} F_{1}\left(\begin{array}{c}
\alpha_{\lambda}, 1-\alpha_{\lambda} \\
2
\end{array} \mid-\frac{x}{\mu^{2}}\right),
$$

where we have used the Gauss recursion formula [9, eq. 9.137.7] for hypergeometric functions. Finally, we note that

$$
\int_{0}^{\infty} \frac{d t \varrho_{\lambda}(t)}{\left(t+\mu^{2}\right)^{3}}=\lim _{x \rightarrow 0} \frac{x-\varrho_{\lambda}(x)}{\lambda x^{2}}=\frac{\alpha_{\lambda}\left(1-\alpha_{\lambda}\right)}{2 \lambda \mu^{2}}=\frac{1}{2 c_{\lambda} \mu^{2}} .
$$

Thus choosing $\mu^{2}=\frac{\alpha_{\lambda}\left(1-\alpha_{\lambda}\right)}{\lambda}$ we confirm (3.15) exactly. 


\section{Stieltjes transform of the measure function}

We find it interesting to directly check that the hypergeometric function $\tilde{\varrho}_{\lambda}(x)=\frac{1}{\mu^{2}} \phi\left(\frac{x}{\mu^{2}}\right)$, see (2.7), solves the integral equation (2.1). The hypergeometric function can be expressed through the more general Meijer-G function. A Meijer-G function is defined by

$$
G_{p, q}^{m, n}\left(z \mid \begin{array}{l}
a_{1}, \ldots, a_{p} \\
b_{1}, \ldots, b_{q}
\end{array}\right)=\frac{1}{2 \pi \mathrm{i}} \int_{L} \frac{\prod_{j=1}^{m} \Gamma\left(b_{j}-s\right) \prod_{j=1}^{n} \Gamma\left(1-a_{j}+s\right)}{\prod_{j=m+1}^{q} \Gamma\left(1-b_{j}+s\right) \prod_{j=n+1}^{p} \Gamma\left(a_{j}-s\right)} z^{s} d s,
$$

with $m, n, p, q \in \mathbb{N}$, with $m \leq q$ and $n \leq p$, and poles of $\Gamma\left(b_{j}-s\right)$ different from poles of $\Gamma\left(1-a_{j}+s\right)$. The infinite contour $L$ separates between the poles of $\Gamma\left(b_{j}-s\right)$ and $\Gamma\left(1-a_{j}+s\right)$, and its behavior to infinity depends on $m, n, p, q$ (see [9, section 9.3]).

The Meijer-G function has by definition the property

$$
G_{p, q}^{m, n}\left(z \mid \begin{array}{l}
a_{1}, \ldots, a_{p} \\
b_{1}, \ldots, b_{q}
\end{array}\right)=\frac{1}{z} G_{q, p}^{n, m}\left(\frac{1}{z} \mid \begin{array}{l}
-b_{1}, \ldots,-b_{q} \\
-a_{1}, \ldots,-a_{p}
\end{array}\right) .
$$

It obeys the convolution formula $[9$, eq. 7.811 .1$]$

$$
\begin{aligned}
& \int_{0}^{\infty} d x G_{p, q}^{m, n}\left(\alpha x \mid \begin{array}{l}
a_{1}, \ldots, a_{p} \\
b_{1}, \ldots, b_{q}
\end{array}\right) G_{p^{\prime}, q^{\prime}}^{m^{\prime}, n^{\prime}}\left(\beta x \mid \begin{array}{l}
a_{1}^{\prime}, \ldots, a_{p^{\prime}}^{\prime} \\
b_{1}^{\prime}, \ldots, b_{q^{\prime}}^{\prime}
\end{array}\right)
\end{aligned}
$$

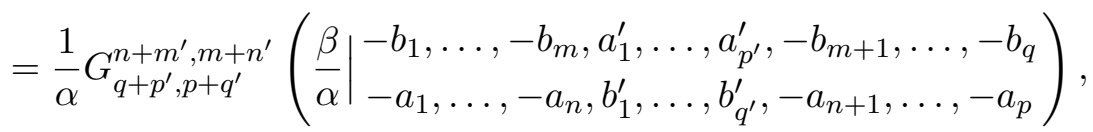

which is the source of numerous impressive integrals over $\mathbb{R}_{+}$of products of special functions. If no two $b_{j}$ differ by an integer, either $p<q$ or $p=q$ with $|z|<1$, then a Meijer-G function can be expressed by hypergeometric functions

$$
\begin{aligned}
G_{p, q}^{m, n}\left(z \mid \begin{array}{c}
a_{1}, \ldots, a_{p} \\
b_{1}, \ldots, b_{q}
\end{array}\right)= & \sum_{k=1}^{m} \frac{\prod_{j=1}^{\prime m} \Gamma\left(b_{j}-b_{k}\right) \prod_{j=1}^{n} \Gamma\left(1+b_{k}-a_{j}\right)}{\prod_{j=m+1}^{q} \Gamma\left(1+b_{k}-b_{j}\right) \prod_{j=n+1}^{p} \Gamma\left(a_{j}-b_{k}\right)} z^{b_{k}} \\
& \times{ }_{p} F_{q-1}\left(\begin{array}{c}
1+b_{k}-a_{1}, \ldots, 1+b_{k}-a_{p} \\
1+b_{k}-b_{1}, \ldots, \star, \ldots, 1+b_{k}-b_{q}
\end{array} \mid(-1)^{p-n-m} z\right),
\end{aligned}
$$

where primed product and the $\star$ means that the term with $j=k$ is omitted.

We need another identity which is derived directly from the definition

$$
\begin{aligned}
& G_{3,3}^{3,2}\left(z \mid \begin{array}{c}
0,0,1 \\
b_{1}, b_{2}, 0
\end{array}\right)=\frac{1}{2 \pi \mathrm{i}} \int_{L} \frac{\Gamma\left(b_{1}-s\right) \Gamma\left(b_{2}-s\right) \Gamma(-s) \Gamma(1+s)^{2}}{\Gamma(1-s)} z^{s} d s \\
& =-\frac{1}{2 \pi \mathrm{i}} \int_{L} \Gamma\left(b_{1}-s\right) \Gamma\left(b_{2}-s\right) \Gamma(s) \Gamma(1+s) z^{s} d s \\
& =\Gamma\left(b_{1}\right) \Gamma\left(b_{2}\right)-\frac{1}{2 \pi \mathrm{i}} \int_{L^{\prime}} \Gamma\left(b_{1}-s\right) \Gamma\left(b_{2}-s\right) \Gamma(s) \Gamma(1+s) z^{s} d s \\
& =\Gamma\left(b_{1}\right) \Gamma\left(b_{2}\right)-G_{2,2}^{2,2}\left(z \mid \begin{array}{c}
0,1 \\
b_{1}, b_{2}
\end{array}\right),
\end{aligned}
$$

where the contour is changed $L \rightarrow L^{\prime}$ such that it is moved through $s=0$ and picked up the residue. The contour $L^{\prime}$ fulfills the definition (4.1) for $G_{2,2}^{2,2}\left(z \mid \begin{array}{c}0,1 \\ b_{1}, b_{2}\end{array}\right)$. 
From (4.4) one can establish

$$
\tilde{\varrho}_{\lambda}(t)=\frac{1}{\mu^{2}} \frac{1}{\Gamma\left(2-\alpha_{\lambda}\right) \Gamma\left(1+\alpha_{\lambda}\right)} G_{2,2}^{1,2}\left(\frac{t}{\mu^{2}} \mid \begin{array}{c}
\alpha_{\lambda}-1,-\alpha_{\lambda} \\
0,-1
\end{array}\right),
$$

and $\frac{1}{x+t+\mu^{2}}=\frac{1}{x+\mu^{2}} 1 F_{0}\left(\begin{array}{c}1 \\ -\end{array} \mid-\frac{t}{x+\mu^{2}}\right)=\frac{1}{x+\mu^{2}} G_{1,1}^{1,1}\left(\left.\frac{t}{x+\mu^{2}}\right|_{0} ^{0} 0\right.$. The convolution theorem (4.3) of Meijer-G functions thus allows to evaluate the integral

$$
\begin{aligned}
& \lambda \int_{0}^{\infty} \frac{d t \tilde{\varrho}_{\lambda}(t)}{x+t+\mu^{2}}
\end{aligned}
$$

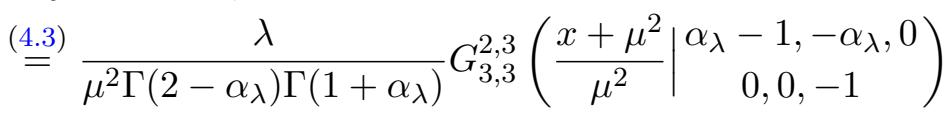

$$
\begin{aligned}
& \stackrel{(4.2)}{=} \frac{\lambda}{\left(x+\mu^{2}\right) \Gamma\left(2-\alpha_{\lambda}\right) \Gamma\left(1+\alpha_{\lambda}\right)} G_{3,3}^{3,2}\left(\frac{\mu^{2}}{x+\mu^{2}} \mid \begin{array}{c}
0,0,1 \\
1-\alpha_{\lambda}, \alpha_{\lambda}, 0
\end{array}\right) \\
& \stackrel{(4.5)}{=} \frac{\lambda}{\left(x+\mu^{2}\right) \Gamma\left(2-\alpha_{\lambda}\right) \Gamma\left(1+\alpha_{\lambda}\right)}\left(\Gamma\left(1-\alpha_{\lambda}\right) \Gamma\left(\alpha_{\lambda}\right)-G_{2,2}^{2,2}\left(\left.\frac{\mu^{2}}{x+\mu^{2}}\right|_{1-\alpha_{\lambda}, \alpha_{\lambda}} \begin{array}{c}
0,1 \\
1-\mu^{2}
\end{array}\right)\right) \\
& \stackrel{(4.4)}{=} \frac{\lambda}{\left(x+\mu^{2}\right)}\left\{\frac{1}{\alpha_{\lambda}\left(1-\alpha_{\lambda}\right)}\right. \\
& -\frac{\Gamma\left(2 \alpha_{\lambda}-1\right) \Gamma\left(1-\alpha_{\lambda}\right)}{\Gamma\left(1+\alpha_{\lambda}\right)}\left(\frac{\mu^{2}}{x+\mu^{2}}\right)^{1-\alpha_{\lambda}}{ }_{2} F_{1}\left(\begin{array}{c}
2-\alpha_{\lambda}, 1-\alpha_{\lambda} \\
2-2 \alpha_{\lambda}
\end{array} \mid \frac{\mu^{2}}{x+\mu^{2}}\right) \\
& \left.-\frac{\Gamma\left(1-2 \alpha_{\lambda}\right) \Gamma\left(\alpha_{\lambda}\right)}{\Gamma\left(2-\alpha_{\lambda}\right)}\left(\frac{\mu^{2}}{x+\mu^{2}}\right)^{\alpha_{\lambda}}{ }_{2} F_{1}\left(\begin{array}{c}
1+\alpha_{\lambda}, \alpha_{\lambda} \\
2 \alpha_{\lambda}
\end{array} \mid \frac{\mu^{2}}{x+\mu^{2}}\right)\right\} \\
& =\frac{1}{\left(x+\mu^{2}\right)} \frac{\lambda}{\alpha_{\lambda}\left(1-\alpha_{\lambda}\right)}-\frac{\lambda \pi}{\sin \left(\alpha_{\lambda} \pi\right)} \tilde{\varrho}_{\lambda}(x) \text {. }
\end{aligned}
$$

We have used the expansion of a Meijer-G function into hypergeometric functions and applied in the last step [9, eq. 9.132.1]. The result is precisely (2.1) provided that $c_{\lambda}=$ $\frac{\lambda}{\alpha_{\lambda}\left(1-\alpha_{\lambda}\right)}($ see $(2.7))$ and $\sin \left(\alpha_{\lambda} \pi\right)=\lambda \pi($ see $(2.8))$.

\section{Outlook}

With the identification of $J$ we have completed the solution of the planar 2-point function of the $\Phi^{4}$-QFT model on four-dimensional Moyal space at the self-duality point. From the 2-point function one directly builds all planar correlation functions [7, 15]. In our earlier work [16] on the much simpler cubic Kontsevich-like model we gave an algorithm to compute also all non-planar correlation functions from the planar sector. It remains to be seen whether a similar endeavour can succeed for the $\Phi^{4}$-model, too.

We expect that non-planar functions are expressed in terms of the inverse function $J^{-1}$. Inverses of hypergeometric functions do not seem to be studied. There is now strong motivation to try it. Of course one can approximate $J^{-1}$ perturbatively via the expansion of $J$ into hyperlogarithms which we established. A non-perturbative characterisation of $J^{-1}$ could provide useful identities between these number-theoretic functions. 


\section{Acknowledgments}

This work was supported by the Erwin Schrödinger Institute (Vienna) through a "Research in Team" grant and by the Deutsche Forschungsgemeinschaft via the Cluster of Excellence ${ }^{1}$ "Mathematics Münster" and the RTG 2149.

\section{A On the spectrum of the integral operator (by Robert Seiringer)}

Abstractly, the integral equation (2.1) is of the form

$$
\psi=f_{\mu}-\lambda A_{\mu} \psi
$$

where $\psi(t)=\tilde{\varrho}_{\lambda}(t), f_{\mu}(t)=\left(t+\mu^{2}\right)^{-1}$ and $A_{\mu}$ is the operator with integral kernel

$$
A_{\mu}(t, u)=\frac{u t}{\left(u+\mu^{2}\right)\left(u+t+\mu^{2}\right)\left(t+\mu^{2}\right)} .
$$

Note that $A_{\mu}$ is symmetric and positive. The equation can thus be solved for $\psi$ if $\lambda>\lambda_{c}=$ $-\left\|A_{\mu}\right\|^{-1}$.

By scaling, the spectrum of $A_{\mu}$ is independent of $\mu$ for $\mu>0$. We claim that

$$
\left\|A_{\mu}\right\|=\left\|A_{0}\right\|=\pi
$$

In particular, $\lambda_{c}=-1 / \pi$.

Since $A_{\mu}$ has a positive kernel which is monotone in $\mu$, one readily obtains $\left\|A_{\mu}\right\| \leq$ $\left\|A_{0}\right\|$. On the other hand, $A_{0}$ is the weak limit of $A_{\mu}$ as $\mu \rightarrow 0$, hence $\left\|A_{0}\right\| \leq$ $\liminf _{\mu \rightarrow 0}\left\|A_{\mu}\right\|$, which proves that $\left\|A_{\mu}\right\|=\left\|A_{0}\right\|$. Now $A_{0}(t, u)=(u+t)^{-1}$. Introducing logarithmic coordinates, we have

$$
\begin{aligned}
\int_{0}^{\infty} \int_{0}^{\infty} \frac{\phi(u)^{*} \phi(t)}{u+t} d u d t & =\int_{\mathbb{R}} \int_{\mathbb{R}} \frac{\phi^{*}\left(e^{v}\right) \phi\left(e^{s}\right)}{e^{v}+e^{s}} e^{v+s} d v d s \\
& =\int_{\mathbb{R}} \int_{\mathbb{R}} \frac{\phi^{*}\left(e^{v}\right) e^{v / 2} \phi\left(e^{s}\right) e^{s / 2}}{2 \cosh \left(\frac{1}{2}(v-s)\right)} d v d s
\end{aligned}
$$

which can be diagonalised via Fourier transforms. Since

$$
\int_{\mathbb{R}} \frac{1}{2 \cosh (v / 2)} d v=\pi
$$

this shows that the spectrum of $A_{0}$ equals $[0, \pi]$, and indeed $\left\|A_{0}\right\|=\left\|A_{\mu}\right\|=\pi$.

Open Access. This article is distributed under the terms of the Creative Commons Attribution License (CC-BY 4.0), which permits any use, distribution and reproduction in any medium, provided the original author(s) and source are credited.

\footnotetext{
1 "Gefördert durch die Deutsche Forschungsgemeinschaft (DFG) im Rahmen der Exzellenzstrategie des Bundes und der Länder EXC 2044 - 390685587, Mathematik Münster: Dynamik-Geometrie-Struktur”.
} 


\section{References}

[1] W.E. Thirring, A soluble relativistic field theory?, Annals Phys. 3 (1958) 91 [INSPIRE].

[2] J.S. Schwinger, Gauge invariance and mass. 2., Phys. Rev. 128 (1962) 2425 [InSPIRE].

[3] D.J. Gross and A. Neveu, Dynamical symmetry breaking in asymptotically free field theories, Phys. Rev. D 10 (1974) 3235 [inSPIRE].

[4] H. Grosse and R. Wulkenhaar, Renormalisation of $\phi^{4}$ theory on noncommutative $\mathbb{R}^{4}$ in the matrix base, Commun. Math. Phys. 256 (2005) 305 [hep-th/0401128] [INSPIRE].

[5] M. Disertori, R. Gurau, J. Magnen and V. Rivasseau, Vanishing of beta function of non commutative $\Phi_{4}^{4}$ theory to all orders, Phys. Lett. B 649 (2007) 95 [hep-th/0612251] [INSPIRE].

[6] E. Langmann and R.J. Szabo, Duality in scalar field theory on noncommutative phase spaces, Phys. Lett. B 533 (2002) 168 [hep-th/0202039] [INSPIRE].

[7] H. Grosse and R. Wulkenhaar, Self-dual noncommutative $\phi^{4}$-theory in four dimensions is a non-perturbatively solvable and non-trivial quantum field theory, Commun. Math. Phys. 329 (2014) 1069 [arXiv: 1205. 0465] [INSPIRE].

[8] H. Grosse, A. Hock and R. Wulkenhaar, Solution of all quartic matrix models, arXiv: 1906.04600 [INSPIRE].

[9] I.S. Gradshteyn and I.M. Ryzhik, Table of integrals, series, and products, seventh ed., Elsevier/Academic Press, Amsterdam, The Netherlands (2007).

[10] S. Ponnusamy and M. Vuorinen, Asymptotic expansions and inequalities for hypergeometric function, Mathematika 44 (1997) 278.

[11] F. Tricomi, Integral Equations, Dover Publications, New York, U.S.A., (1985).

[12] E. Panzer and R. Wulkenhaar, Lambert-W solves the noncommutative $\Phi^{4}$-model, Commun. Math. Phys. (2019) [arXiv:1807.02945] [INSPIRE].

[13] F.C.S. Brown, Multiple zeta values and periods of moduli spaces $\mathcal{M}_{0, n}$, Annales Sci. Ecole Norm. Sup. 42 (2009) 371 [math/0606419].

[14] E. Panzer, Algorithms for the symbolic integration of hyperlogarithms with applications to Feynman integrals, Comput. Phys. Commun. 188 (2015) 148 [arXiv:1403.3385] [INSPIRE].

[15] J. de Jong, A. Hock and R. Wulkenhaar, Catalan tables and a recursion relation in noncommutative quantum field theory, arXiv:1904.11231.

[16] H. Grosse, A. Hock and R. Wulkenhaar, A Laplacian to compute intersection numbers on $\overline{\mathcal{M}}_{g, n}$ and correlation functions in NCQFT, arXiv:1903.12526 [INSPIRE]. 